\title{
GMR
}

\section{Effect of procyanidine on VEGFR-2 expression and transduction pathway in rat endothelial progenitor cells under high glucose conditions}

\author{
Y. Liu*, W.-J. Liao*, Z. Zhu*, H. Zeng, H.-Q. He, X.-L. Sun, X.-F. Xu, L. Huang, \\ W.-M. Wang, X.-Y. Zhou and Y.-Z. He \\ Department of Vascular Surgery, Affiliated Hospital of Luzhou Medical College, \\ Jiang Yang District, Luzhou, Sichuan, China \\ *These authors contributed equally to this study. \\ Corresponding authors: Y.-Z. He / Y. Liu \\ E-mail: heyanzheng2012@163.com / lyong74@163.com
}

Genet. Mol. Res. 15 (1): gmr.15016925

Received August 10, 2015

Accepted October 30, 2015

Published March 31, 2016

DOI http://dx.doi.org/10.4238/gmr.15016925

\begin{abstract}
The protective effect of procyanidine and its oligomers against high glucose-mediated oxidative stress injury in endothelial progenitor cells (EPCs), and effect of procyanidin on vascular endothelial growth factor receptor-2 (VEGFR-2) expression and downstream signal pathway were analyzed in vitro. Rat bone marrow mononuclear cells were isolated, cultured under normal and high glucose (HG) conditions, and the changes in cell morphology observed. The EPCs were identified, and the oxidative stress products produced by EPCs (under normal and HG conditions) were quantified. Subsequently, an appropriate number of EPCs were cultured with and without procyanidin (OPC), and the MDA concentration and relative expression of VEGFR-2, AKT, IkB- $\alpha$, and nuclear factor (NF)-kB were detected 1, 3, 5, and 7 days post-culture. We observed minor (round, translucent, gradually adhering) and significant (fusiform morphology/ pebble distribution) cell morphological changes 3 and 7 days post-culture,
\end{abstract}


respectively. Apoptosis and oxidative stress product release in EPCs cultured with HG increased significantly compared to the control group ( $P$ $<0.05)$. The oxidative stress product generation and relative expression of VEGFR-2, AKT, I $\mathrm{KB}-\alpha$, and NF- $\kappa \mathrm{B}$ were not significantly affected by OPC addition in normal glucose conditions ( $P>0.05)$; alternately, products generated as a result of oxidative stress were significantly reduced, the relative expression of VEGFR-2, AKT, and NF- $\mathrm{KB}$ protein was upregulated, and that of $I_{\kappa} \mathrm{B}-\alpha$ was downregulated $(P<0.05)$ in $\mathrm{HG}+\mathrm{OPC}$ EPCs. Therefore, procyanidin may promote cell proliferation by alleviating oxidative damage to EPCs under HG conditions, and upregulating VEGFR-2 expression and its downstream signal pathway.

Key words: Procyanidine; Progenitor cells; High sugar concentration; Oxidative stress; VEGFR-2

\section{INTRODUCTION}

Diabetes mellitus is a metabolic disorder, with features including high blood sugar and insulin deficiency-induced impaired glucose tolerance and/or islet dysfunction (Brice et al., 2015). Diabetic vascular disease is the leading cause of death and disability in diabetic patients; $30-40 \%$ of the patients are subject to at least one vascular complications after 10 years (Tavintharan et al., 2014). Numerous studies have shown the association of diabetes mellitus vascular complications with oxidative stress in endothelial cells and endothelial cells derived from progenitor cells (mainly in the bone marrow) (Kizub et al., 2014; Prieto et al., 2014; Yiu and Tse, 2014). Conventionally, high blood sugar is believed to lead to microvascular and macrovascular disease via four pathways: 1) polyol signaling pathway imbalances, 2) increase in advanced glycation end-product (AGE) formation, 3) increased activation of protein kinase $\mathrm{C}$; and 4) imbalances in the hexosamine signaling pathway (van den Oever et al., 2010).

In recent years, the possible role of high glucose concentrations in inducing the pathogenesis of diabetic vascular complications has been reported by a number of studies; high glucose concentrations affect the mitochondrial electron transport chain, leading to the "escape" of internal electronic mechanisms, and the generation of large amounts of reactive oxygen species (ROS) (Yao et al., 2006; Hamed et al., 2009). The accumulation of ROS in vivo in concentrations that significantly exceed the cell mass (which are at clear range) causes oxidative stress injury, which in turn affects the cell growth and survival cycle. In fact, this is responsible for abnormal metabolism and dysfunction in cells, as well as apoptosis. Therefore, several researchers are attempting to identify effective antioxidant drugs to relieve oxidative stress injury in diabetic vascular complications. A large number of studies have stratified vascular endothelial growth factor receptors (VEGFR) into three subtypes; among these, VEGFR-2 is mainly distributed in the lymphatic and vascular endothelial cell membrane. Studies have also shown that endothelial progenitor cells (EPCs) express VEGFR-2, and that VEGFR-2 activation regulates cell proliferation. In this study, we attempted to elucidate the protective effect of procyanidin and its oligomers on EPCs against oxidative stress. Procyanidin is an antioxidant present in most plants, with the highest content observed in berry plants (Loomans et al., 2005). Studies have indicated that an increase in the consumption of fruits and vegetables may lead to a corresponding decrease in the incidence of cardiovascular disease. A large number of vegetables and fruits are rich in anthocyanins; grape 
seeds have the highest concentration of anthocyanin among these. The anti-inflammatory and antioxidant ability of anthocyanin has been substantially proven; eating foods rich in anthocyanin have been reported to stabilize blood pressure and prevent the occurrence of cardiovascular disease (Ghafoor et al., 2009). Recent studies have shown that anthocyanins protect against and repair endothelial dysfunction induced by the release of oxidative stress products, and reduce the risk factors for cardiovascular disease. Long-term intake of proanthocyanidin and anthocyanin-rich fruits can significantly reduce the risk of type 2 diabetes (Wedick et al., 2012; Hassellund et al., 2013).

In this study, oxidative stress induced by high glucose was reduced by the administration of proanthocyanidin, in order to verify the role of ROS in VEGFR signaling pathway, which regulates endothelial progenitor cell proliferation and maintains the integrity of the structure and function of endothelial progenitor cells. At present, very few studies conducted domestically and internationally have reported on the regulatory mechanism of oxidative stress under high glucose conditions in EPCs; therefore, in this study, we have attempted to elucidate the effect of proanthocyanidins on the antioxidant function of EPCs exposed to high glucose for the first time. Additionally, we have determined the VEGFR-2 concentration on EPC membrane before and after proanthocyanin exposure, in order to open new avenues for the treatment of diabetic vascular complications.

\section{MATERIAL AND METHODS}

\section{Experimental animals}

Forty healthy adult Sprague-Dawley rats (average age: 4 months; animal grade: 2) weighing $200 \pm 10 \mathrm{~g}$ were provided by the Experimental Animal Center of Luzhou Medical College (No. 24101115).

\section{EPC isolation, culture, and identification}

EPCs were isolated, cultured, and identified using methods previously reported in literature (Liu et al., 2015).

\section{CCK-8 assay for the detection of EPC proliferation under varying glucose concentrations}

EPCs were divided into two groups: the normal glucose concentration group (Con, $5.5+$ $25 \mathrm{mM}$ mannitol) and high glucose concentration group (HG, $30 \mathrm{mM}$ glucose). EPCs were digested and seeded on two 96-well plates at a density of $2 \times 10^{5}$ cells $/ \mathrm{mL}$. One hundred microliters of the cell suspension was added to five wells on each plate at each of four time points $(1,3,5,7$ days). Ten microliters of a standard CCK-8 reagent was added to the cells from each time point cultured for 1, 3, 5, and 7 days. Optical densities (OD) of the cells at $450 \mathrm{~nm}$ were detected using a microplate reader after culturing for $1.5 \mathrm{~h}$.

\section{Detection of MDA concentration in EPCs cultured with different glucose concentrations}

EPCs were cultured on 6-well plates, and digested 1, 3, 5, and 7 days later. The EPC density was adjusted to $2 \times 10^{5}$ cells $/ \mathrm{mL}$ in each well, transferred to freezing tubes, and incubated 
at $-20^{\circ} \mathrm{C}$ for $1 \mathrm{~h}$. The tubes were then thawed at room temperature. This was repeated for 3 cycles. A cell homogenate was prepared by lysing the cells. MDA concentrations were measured using a standard kit, according to the manufacturer instructions.

\section{Screening for optimal proanthocyanidin (OPC) concentration}

EPCs in the HG group were digested and seeded to eight 96-well plates at a density of $2 \times 10^{5} \mathrm{cells} / \mathrm{mL}$ per well. Five wells on each plate were assigned to one of four time points, and $100 \mu \mathrm{L}$ cell suspension was added to the wells at each time point. OPC was added at the following concentrations: $0,10,15,20,25,30,35$, and $40 \mu \mathrm{g} / \mathrm{mL}$, to the wells of an 8-well plate. The cells were divided into 8 groups, and CCK-8 reagent $(10 \mu \mathrm{L})$ was added to each of these cell groups at $1,3,5$, and 7 days post-culture. The OD at $450 \mathrm{~nm}$ was then determined using a microplate reader after $1.5 \mathrm{~h}$.

\section{MDA concentration in EPCs after OPC intervention}

EPCs assigned to four groups (Con, Con + OPC, HG + OPC, and HG) were cultured for 1, 3,5 , and 7 days; subsequently, the MDA concentrations in these cells were measured, as detailed by the MDA kit manufacturers.

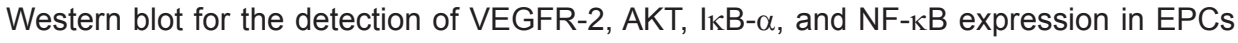
were seeded on 96-well plates. The cells were divided into four groups: Con, Con + OPC, HG + OPC, and HG, based on the type of supplement. The total protein produced by EPCs on each plate were extracted $1,3,5$, and 7 days post-culture: the cells were centrifuged, the supernatant was collected, and the cell debris removed. Protein concentration was determined using the bicinchoninic acid method. The extracted proteins $(2 \mu \mathrm{g} / \mu \mathrm{L})$ were then electrophoresed and transferred to an NC membrane. The membrane was blocked for $2 \mathrm{~h}$ at $37^{\circ} \mathrm{C}$, and subsequently incubated overnight with rabbit anti-mouse VEGFR-2 primary antibody at $4^{\circ} \mathrm{C}$ on a shaker. The membrane was then washed four times with PBST. The membrane was developed using an appropriate developing solution for $3 \mathrm{~min}$, and subsequently rinsed with distilled water to terminate the reaction. The membrane was scanned; subsequently, the molecular weight of the target band was determined and the net OD of the bands measured using a gel image processing system.

\section{Matrigel for the detection of tube number in each group}

Matrigel basement membrane matrix is an extracellular matrix protein chiefly comprising laminin and type IV collagen extracted from mouse tumor cells. High concentrations of the Matrigel matrix are used to create a three-dimensional Matrigel at $22^{\circ}-35^{\circ} \mathrm{C}$, which can be used to study cell morphology, function, and differentiation. The Matrigel gradually assumes a tube-like structure when used in the study of differentiation of endothelial progenitor cells into mature endothelial cells. Matrigel basement membrane matrix simulates the biological functions of cell basement membrane in vivo to provide a suitable environment for angiogenesis.

Matrigel was mixed with serum-free media at a ratio of $1: 1$; the mixture was spread on a 24-well plate, and incubated for half an hour in order to promote solidification. EPCs were digested and divided into four groups: Con $+30 \mu \mathrm{g} / \mathrm{mL}$ OPC, Con, $\mathrm{HG}+30 \mu \mathrm{g} / \mathrm{mL} \mathrm{OPC}$, and the HG groups. Each well contained $2 \times 10^{4}$ cells. The plates were cultured for $24 \mathrm{~h}$, and the number of tubes in three randomly selected horizons were counted. 


\section{Statistical analysis}

Experimental $O D$ values and relative protein expression levels are reported as means \pm standard deviation (SD). The data were analyzed using SPSS v.13.0 (IBM, Armonk, NY, USA). Comparisons between the Con and HG groups, Con + OPC and Con groups, and HG + OPC and HG groups were analyzed using the $t$-test.

\section{RESULTS}

\section{Morphological changes in EPCs and double-swallow staining}

A majority of the EPCs were round and translucent, and had gradually begun to adhere after 3 days after seeding to 6-well plates (Figure 1A). At 7 days, EPCs showed significant morphological changes. A majority of the cells displayed a fusiform morphology and gathering growth (Figure 1B). At 14 days post-inoculation, the cells were big and round with increasing transparency, showing a "pebble" form distribution (Figure 1C). The EPCs were treated with Dil-acLDL and FITC-UEA-1, and the cell color determined by confocal microscopy. FITC-UEA-1-treated (Figure 2A) and Dil-ac-LDL-treated (Figure 2B) EPCs showed a green and red fluorescence, respectively. Blue fluorescence denoted nuclear staining (Figure 2C). Differentiating EPCs showed double green and red staining (Figure 2D).

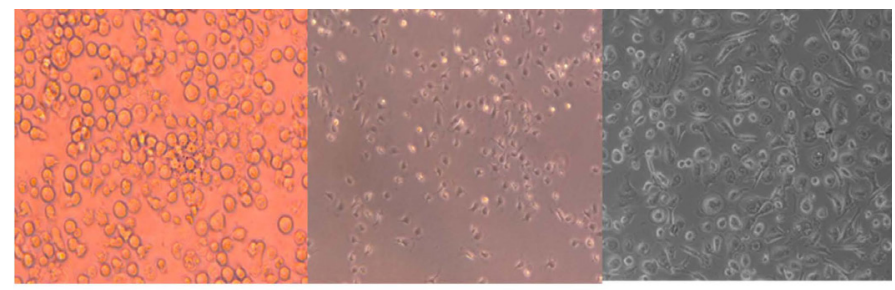

A

B

C

Figure 1. Morphological changes in endothelial progenitor cells at different time points. A. 3 days; B. 7 days; C. 14 days.

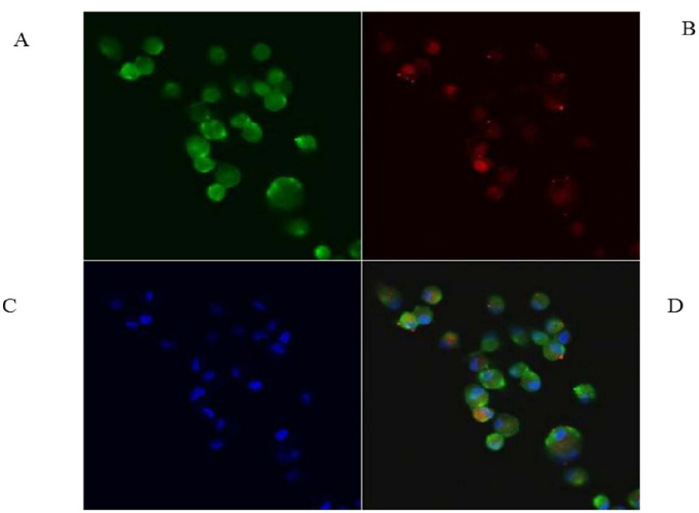

Figure 2. Endothelial progenitor cells were identified by Dil-ac-LDL and FITC-UEA-1 double staining. A. FITC-UEA-1; B. Dil-ac-LDL; C. DAPI; and D. Merge. 


\section{EPC proliferation under different glucose concentrations}

The OD of EPCs in the Con group increased gradually at days 1, 3, 5, and 7. Alternately, the OD values of EPCs cultured under high glucose conditions decreased gradually with time. The OD values shown by EPCs in the Con group were significantly higher than those shown by EPCs in the HG group ( $P<0.05$; Table 1$)$.

Table 1. CCK-8 testing of endothelial progenitor cell proliferation under different glucose concentrations.

\begin{tabular}{l|c|c|c|c}
\hline Groups & 1 day & 3 days & 5 days & 7 days \\
\hline Normal glucose concentration & $0.76 \pm 0.03^{*}$ & $0.92 \pm 0.03^{*}$ & $1.50 \pm 0.16^{*}$ & $2.25 \pm 0.30^{*}$ \\
\hline High glucose concentration & $0.37 \pm 0.03$ & $0.32 \pm 0.01$ & $0.24 \pm 0.03$ & $0.18 \pm 0.03$ \\
\hline
\end{tabular}

Compared to the high glucose group, ${ }^{*} \mathrm{P}<0.05$.

\section{MDA concentration of EPCs under differing glucose concentrations}

MDA concentrations of EPCs in the Con group increased non-significantly with time. However, the MDA concentrations in the HG EPCs increased significantly compared to the Con EPCs $(P<0.05 ;$ Table 2).

Table 2. Changes in MDA value in endothelial progenitor cells cultured under different glucose concentrations.

\begin{tabular}{l|c|c|c|c}
\hline Groups & 1 day & 3 days & 5 days & 7 days \\
\hline Normal glucose concentration & $0.66 \pm 0.02^{*}$ & $0.71 \pm 0.01^{*}$ & $0.70 \pm 0.02^{*}$ & $0.69 \pm 0.01^{*}$ \\
\hline High glucose concentration & $1.28 \pm 0.20$ & $3.44 \pm 0.16$ & $4.02 \pm 0.19$ & $4.58 \pm 0.16$ \\
\hline
\end{tabular}

Compared to the high glucose group, ${ }^{*} \mathrm{P}<0.05$.

\section{Determination of appropriate procyanidine concentration for optimal screening}

At each time point, obvious EPC proliferation was observed at an OPC concentration of $30 \mu \mathrm{g} /$ $\mathrm{mL}$. At a specific time point, the OD of EPCs treated with $30 \mu \mathrm{g} / \mathrm{mL}$ OPC was significantly higher $(\mathrm{P}<$ 0.05 ) than that of EPCs treated with $25 \mu \mathrm{g} / \mathrm{mL}$ OPC, and did not differ significantly $(P>0.05)$ compared to EPCs treated with $35 \mu \mathrm{g} / \mathrm{mL}$ OPC. This indicated that EPC proliferation was obvious at an OPC concentration of $30 \mu \mathrm{g} / \mathrm{mL}$, and this concentration was used in all subsequent experiments (Table 3 ).

Table 3. EPC proliferation in the presence of procyanidin.

\begin{tabular}{l|c|c|c|c|c|c|c|c}
\hline Time point & $0 \mu \mathrm{g} / \mathrm{mL}$ & $10 \mu \mathrm{g} / \mathrm{mL}$ & $15 \mu \mathrm{g} / \mathrm{mL}$ & $20 \mu \mathrm{g} / \mathrm{mL}$ & $25 \mu \mathrm{g} / \mathrm{mL}$ & $30 \mu \mathrm{g} / \mathrm{mL}$ & $35 \mu \mathrm{g} / \mathrm{mL}$ & $40 \mu \mathrm{g} / \mathrm{mL}$ \\
\hline 1 day & $0.29 \pm 0.02$ & $0.32 \pm 0.03$ & $0.34 \pm 0.04$ & $0.36 \pm 0.01$ & $0.36 \pm 0.02$ & $0.46 \pm 0.01^{*}$ & $0.44 \pm 0.02$ & $0.42 \pm 0.02$ \\
\hline 3 days & $0.31 \pm 0.01$ & $0.31 \pm 0.02$ & $0.36 \pm 0.02$ & $0.37 \pm 0.03$ & $0.37 \pm 0.02$ & $0.43 \pm 0.03^{*}$ & $0.42 \pm 0.01$ & $0.41 \pm 0.03$ \\
\hline 5 days & $0.28 \pm 0.03$ & $0.33 \pm 0.03$ & $0.33 \pm 0.03$ & $0.38 \pm 0.03$ & $0.35 \pm 0.02$ & $0.44 \pm 0.02^{*}$ & $0.42 \pm 0.03$ & $0.41 \pm 0.02$ \\
\hline 7 days & $0.26 \pm 0.03$ & $0.32 \pm 0.01$ & $0.35 \pm 0.03$ & $0.37 \pm 0.02$ & $0.36 \pm 0.01$ & $0.42 \pm 0.02^{*}$ & $0.41 \pm 0.03$ & $0.42 \pm 0.01$ \\
\hline
\end{tabular}

\section{Effect of procyanidine on MDA in EPCs cultured under high glucose concentrations}

The MDA concentration in the EPCs in the Con + OPC and Con groups cultured for 1, 3, 5 , and 7 days did not differ significantly $(P>0.05)$. On the other hand, the MDA concentrations in the EPCs was significantly lower in the HG + OPC group at the four time points compared to the HG group ( $P<0.05$; Table 4). 
Table 4. Change in MDAexpression in endothelial progenitorcells in the presence and absence of procyanidin(OPC).

\begin{tabular}{l|c|c|c|c}
\hline Groups & 1 day & 3 days & 5 days & 7 days \\
\hline Con + OPC & $0.74 \pm 0.02$ & $0.83 \pm 0.02$ & $0.91 \pm 0.01$ & $1.04 \pm 0.06$ \\
\hline Con & $0.74 \pm 0.01$ & $0.85 \pm 0.01$ & $0.89 \pm 0.02$ & $1.06 \pm 0.10$ \\
\hline HG + OPC & $0.85 \pm 0.01^{*}$ & $1.50 \pm 0.02^{*}$ & $1.96 \pm 0.09^{*}$ & $2.19 \pm 0.18^{*}$ \\
\hline HG & $1.25 \pm 0.12$ & $3.25 \pm 0.11$ & $3.55 \pm 0.15$ & $4.56 \pm 0.14$ \\
\hline
\end{tabular}

Con = normal glucose concentration; $\mathrm{HG}=$ high glucose concentration. Compared to the high glucose group, ${ }^{*} \mathrm{P}<0.05$.

\section{Effect of procyanidine on VEGFR-2, AKT, NF-kB, and IкB- $\alpha$ expression under high glucose concentrations}

The relative expressions of the four proteins did not differ significantly at the four time points $(1,3,5$, and 7 days) between the Con + OPC and Con groups $(P>0.05)$. The relative expression of VEGFR-2, AKT, and NF-kB, was increased by $6.20,6.80,7.64$, and 3.58 times $(P<0.05)$; 3.81, 3.46, 5.11, and 6.00 times $(P<0.05) ; 1.75,2.44,3.26$, and 3.46 times $(P<0.05)$; and that of $\mathrm{IkB}-\alpha$ was reduced by $1.41,1.34,1.08$, and 1.09 times $(P<0.05)$ at the four time points $(1,3,5$, and 7 days), respectively, in the HG + OPC group compared to the HG group. The relative expression of VEGFR-2, AKT, and NF-kB/p65 increased gradually with time in the HG + OPC group (Figure 3).

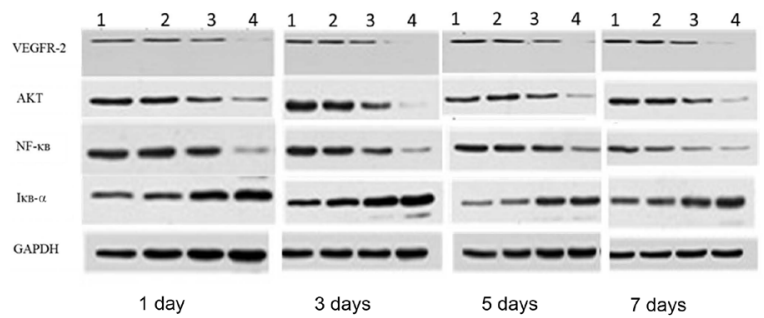

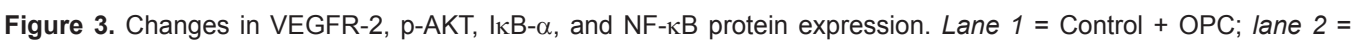
control; lane 3 = HG + OPC; and lane $4=$ HG.

\section{Effect of OPC on tube formation in each group}

The EPC-tube formation did not differ significantly between the normal glucose concentration + OPC and normal glucose concentration groups $(21 \pm 0.45$ vs $20 \pm 0.6$; P > 0.05), while the EPC-Matrigel tubes formed with cells from the HG + OPC and HG groups differed significantly ( $17 \pm 0.73$ vs $10 \pm 0.63$; $\mathrm{P}<0.05$; Figure 4$)$.

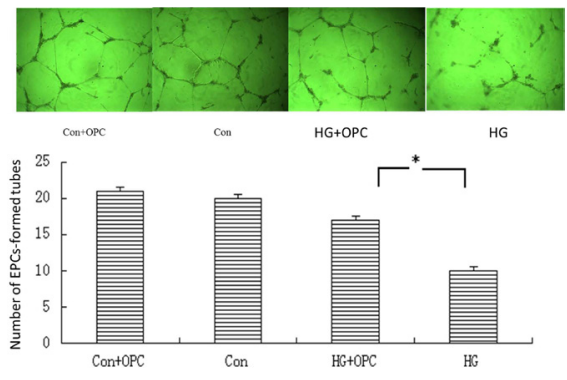

Figure 4. Angiogenesis experiment. * $\mathrm{P}<0.05$ compared to the high glucose group. $\mathrm{Con}=$ normal glucose concentration; $\mathrm{HG}=$ high glucose concentration; $\mathrm{OPC}=$ procyanidin . 


\section{DISCUSSION}

Several studies (Kanikarla-Marie and Jain, 2015; Liu et al., 2015; Seebacher et al., 2015; Wang et al., 2015) have reported that high glucose concentrations lead to over-aggregation of ROS in the body, which results in oxidative stress injury and affects the cell growth and survival cycle. This causes abnormal metabolism and dysfunction in cells, as well as apoptosis, ultimately resulting in diabetic vascular complications, ischemic necrosis, ischemic neuropathy, and paresthesia in the affected limbs. Activation of the polyol signaling pathway and the increase in AGE formation has been strongly associated with oxidative stress (Gradinaru et al., 2012; Tantalaki et al., 2014). Under normal conditions, EPCs grown in the bone marrow are in a state of dormancy; vascular injury, heart failure or myocardial infarction, limb ischemia and hypoxia, or cytokine or drug stimulation induces the migration of EPCs to the lesion, promoting angiogenesis and injury repair in the affected area. Studies have shown that the cells produce an excess of oxidative stress products under high glucose conditions, which is far beyond the elimination and repair capacity of the body (Stadler et al., 2004; Jiang et al., 2012). Excessive accumulation of ROS causes disorders in the normal cell growth cycle, leading to the induction of apoptosis. Currently, several methods, traditionally divided into two types (antioxidant constituents and oxidation products), are being employed to detect the degree of oxidative injury in cells. The increase in oxidative products is correlated with the decrease in the antioxidant constituents of the cells, as the former consumes a considerable amount of antioxidant constituents. Catalase, glutathione reductase, superoxide dismutase, and vitamins are commonly used as indicators of antioxidant constituents. The production of a large number of oxidative stressrelated products in the body leads to the destruction of the antioxidant system balance, which in turn is responsible for the denaturation of lipids, proteins, and DNA. Other indicators have been used to indicate the extent of injury induced by macromolecules such as hydrogen peroxide, superoxide anion, MDA, nitrotyrosine, and $8 \mathrm{OH}$-deoxyguanosine. The MDA content in cells reflects the degree of lipid peroxidation, which is often used to measure the severity of intracellular oxidative stress injury. The results of this study showed that the MDA content generated by EPCs under high glucose conditions was significantly higher than that generated by cells subjected to normal glucose conditions. We also observed a time-dependent increase in MDA concentration, which in turn was an indicator of the increased severity of the oxidative stress injury in EPCs. These results were consistent with those reported in literature; high glucose concentrations significantly inhibited the proliferation of endothelial progenitor cells in a time-dependent manner.

The Con + OPC and Con groups and HG + OPC and HG groups were appointed as two sets of controls for the detection of VEGFR-2, AKT, and NF- $\kappa B$ expression in EPCs at four different time points $(1,3,5$, and 7 days). Procyanidins did not affect the VEGFR-2 expression in EPCs under normal glucose concentrations, while significantly increasing the VEGFR-2 expression and its downstream signaling molecules under high glucose conditions in a time-dependent manner. This provided a theoretical basis for the time-dependent mediation of anti-oxidative stress by proanthocyanidins. NF- $\kappa B$ loses its transcriptional activity in the presence of its inhibitor $I \kappa B$ in the cytoplasm of normal EPCs. AKT activates $I_{\kappa} B$ kinase to promote $I_{\kappa} B$ phosphorylation and

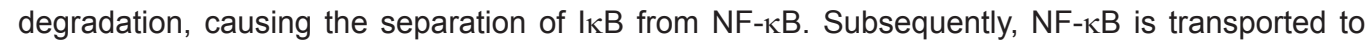
the nucleus, where it regulates transcription, thereby promoting cell proliferation and survival (Liu et al., 2015). We observed no significant difference in the expression of $1 \kappa B-\alpha$ between Con and Con + OPC EPCs (at all time points); on the other hand, $I_{\kappa} \mathrm{B}-\alpha$ expression in the HG + OPC group was significantly lower than that in the HG group, further confirming that proanthocyanidins may alleviate the oxidative damage caused by high glucose in EPCs. 
In summary, high glucose concentrations stimulate the excessive production of oxidative stress products in EPCs, inhibiting EPC proliferation and tube formation. Proanthocyanidins reduce the oxidative stress response of EPCs induced under high glucose conditions, in turn decreasing the damage to EPCs, and improving cell proliferation and tube formation. The latter functions may be achieved through the upregulation of VEGFR-2, AKT, and NF- $\mathrm{BB}$, and the downregulation of $I_{\kappa} B-\alpha$ in EPCs, which promotes the proliferative and tube formation abilities of EPCs. This indicated that these proteins may be key factors affecting EPC function, thereby indicating the presence of a separate pathway for the repair of vascular damage in EPCs in diabetic patients. However, these results must be validated by further animal experiments and clinical trials.

The extent of tube formation and MDA expression in EPCs cultured under normal and high glucose conditions and in the presence or absence of procyanidin was analyzed at different time points, in order to quantify the oxidative stress products produced by the EPCs. The tube numbers and oxidative stress product generation did not differ significantly between the Con and Con + OPC groups, while significant differences were observed (in these values) between the $\mathrm{HG}+\mathrm{OPC}$ and HG groups: the tube number and product of oxidative stress produced in the EPCs of the HG + OPC group were respectively greater and lesser than those observed in the HG group at all time points; additionally, the amount of oxidative stress product in the HG + OPC EPCs appeared to decrease with time. These results indicated that normal glucose conditions do not stimulate the excessive production of oxidative stress products (beyond their clearance ability) in EPCs, causing cell damage, while a high glucose environment significantly induces the generation of oxidative stress, thereby inhibiting EPC proliferation. Additionally, we observed that OPC significantly downregulates the stress (high glucose)-mediated generation of oxidative stress products in EPCs in a time-dependent manner. These results implied that proanthocyanidin intervention relieves the oxidative stress product-mediated inhibition of EPC tube formation and improves blood flow in the affected limbs of diabetic patients with vascular complications, ultimately producing a good clinical effect.

In conclusion, high sugar induces an increase in oxidative stress product concentration in EPCs, impairing EPC function in a time-dependent manner. Procyanidins and their oligomeric products can reduce the oxidative stress response induced by high glucose, thereby reducing EPC injury; this provided experimental evidence for the application of procyanidins to the repair of vascular injury in diabetic patients. The anti-oxidative effect of procyanidins and their oligomeric products could be attributed to the increase in VEGFR-2 expression on the cell membrane of EPCs and the decrease in high glucose-mediated inhibition of EPC proliferation through the activation of downstream pathways. Vascular complications of diabetes could therefore be clinically remedied by antioxidant therapy, which improves the EPC tube formation, promotes angiogenesis at the site of injury, and improves injury repair.

\section{Conflicts of interest}

The authors declare no conflict of interest.

\section{ACKNOWLEDGMENTS}

Research supported by the National Natural Science Foundation of China (\#81270358). 


\section{REFERENCES}

Brice R, Shelley S, Chaturvedi P, Glah D, et al. (2015). Resource use and outcomes associated with initiation of injectable therapies for patients with type 2 diabetes mellitus. Drugs Context 4: 212269.http://dx.doi.org/10.7573/dic.212269

Ghafoor K, Choi YH, Jeon JY and Jo IH (2009). Optimization of ultrasound-assisted extraction of phenolic compounds, antioxidants, and anthocyanins from grape (Vitis vinifera) seeds. J. Agric. Food Chem. 57: 4988-4994.http://dx.doi. org/10.1021/jf9001439

Gradinaru D, Borsa C, lonescu C, Margina D, et al. (2012). Vitamin D status and oxidative stress markers in the elderly with impaired fasting glucose and type 2 diabetes mellitus. Aging Clin. Exp. Res. 24: 595-602.

Hamed S, Brenner B, Aharon A, Daoud D, et al. (2009). Nitric oxide and superoxide dismutase modulate endothelial progenitor cell function in type 2 diabetes mellitus. Cardiovasc. Diabetol. 8: 56.http://dx.doi.org/10.1186/1475-2840-8-56

Hassellund SS, Flaa A, Kjeldsen SE, Seljeflot I, et al. (2013). Effects of anthocyanins on cardiovascular risk factors and inflammation in pre-hypertensive men: a double-blind randomized placebo-controlled crossover study. J. Hum. Hypertens. 27: 100-106.http://dx.doi.org/10.1038/jhh.2012.4

Jiang J, Chen P, Chen J, Yu X, et al. (2012). Accumulation of tissue advanced glycation end products correlated with glucose exposure dose and associated with cardiovascular morbidity in patients on peritoneal dialysis. Atherosclerosis 224: 187194.http://dx.doi.org/10.1016/j.atherosclerosis.2012.06.022

Kanikarla-Marie P and Jain SK (2015). Hyperketonemia (acetoacetate) upregulates NADPH oxidase 4 and elevates oxidative stress, ICAM-1, and monocyte adhesivity in endothelial cells. Cell. Physiol. Biochem. 35: 364-373.http://dx.doi. org/10.1159/000369702

Kizub IV, Klymenko KI and Soloviev Al (2014). Protein kinase C in enhanced vascular tone in diabetes mellitus. Int. J. Cardiol. 174: 230-242.http://dx.doi.org/10.1016/j.jijcard.2014.04.117

Liu ZW, Wang JK, Qiu C, Guan GC, et al. (2015). Matrine pretreatment improves cardiac function in rats with diabetic cardiomyopathy via suppressing ROS/TLR-4 signaling pathway. Acta Pharmacol. Sin. 36: 323-333.http://dx.doi. org/10.1038/aps.2014.127

Loomans CJ, De Koning EJ, Staal FJ, Rabelink TJ, et al. (2005). Endothelial progenitor cell dysfunction in type 1 diabetes: another consequence of oxidative stress? Antioxid. Redox Signal. 7: 1468-1475.http://dx.doi.org/10.1089/ars.2005.7.1468

Prieto D, Contreras C and Sánchez A (2014). Endothelial dysfunction, obesity and insulin resistance. Curr. Vasc. Pharmacol. 12: 412-426.http://dx.doi.org/10.2174/1570161112666140423221008

Seebacher NA, Richardson DR and Jansson PJ (2015). Glucose modulation induces reactive oxygen species and increases P-glycoprotein-mediated multidrug resistance to chemotherapeutics. Br. J. Pharmacol. 172: 2557-2572.http://dx.doi. org/10.1111/bph.13079

Stadler K, Jenei V, Somogyi A and Jakus J (2004). [Aminoguanidine prevents peroxynitrite production and cardiac hypertrophy in streptozotocin-induced diabetic rats]. Orv. Hetil. 145: 2491-2496.

Tantalaki E, Piperi C, Livadas S, Kollias A, et al. (2014). Impact of dietary modification of advanced glycation end products (AGEs) on the hormonal and metabolic profile of women with polycystic ovary syndrome (PCOS). Hormones 13: 65-73.

Tavintharan S, Pek LT, Liu JJ, Ng XW, et al. (2014). Osteoprotegerin is independently associated with metabolic syndrome and microvascular complications in type 2 diabetes mellitus. Diab. Vasc. Dis. Res. 11: 359-362.http://dx.doi. org/10.1177/1479164114539712

van den Oever IA, Raterman HG, Nurmohamed MT and Simsek S (2010). Endothelial dysfunction, inflammation, and apoptosis in diabetes mellitus. Mediators Inflamm. 2010: 792393.http://dx.doi.org/10.1155/2010/792393

Wang C, Blough E, Arvapalli R, Dai X, et al. (2015). Acetaminophen attenuates glomerulosclerosis in obese Zucker rats via ROS/ p38MAPK signaling pathways. Free Radic. Biol. Med. 81: 47-57.http://dx.doi.org/10.1016/j.freeradbiomed.2015.01.008

Wedick NM, Pan A, Cassidy A, Rimm EB, et al. (2012). Dietary flavonoid intakes and risk of type 2 diabetes in US men and women. Am. J. Clin. Nutr. 95: 925-933.http://dx.doi.org/10.3945/ajcn.111.028894

Yao EH, Yu Y and Fukuda N (2006). Oxidative stress on progenitor and stem cells in cardiovascular diseases. Curr. Pharm. Biotechnol. 7: 101-108.http://dx.doi.org/10.2174/138920106776597685

Yiu KH and Tse HF (2014). Specific role of impaired glucose metabolism and diabetes mellitus in endothelial progenitor cell characteristics and function. Arterioscler. Thromb. Vasc. Biol. 34: 1136-1143.http://dx.doi.org/10.1161/ ATVBAHA.114.302192 